\title{
Perfil epidemiológico dos casos de tuberculose no Estado de Pernambuco de 2009 a
}

\section{9}

\author{
Epidemiological profile of tuberculosis cases in the State of Pernambuco from 2009 to 2019 \\ Perfil epidemiológico de los casos de tuberculosis en el Estado de Pernambuco de 2009 a 2019
}

Recebido: 12/02/2021 | Revisado: 21/02/2021 | Aceito: 25/02/2021 | Publicado: 04/03/2021

João Lúcio Macário Lira

ORCID: https://orcid.org/0000-0002-0043-6125 Centro Universitário CESMAC, Brasil E-mail: joaoluciomacario@gmail.com

Maríllia Ferreira Calado

ORCID: https://orcid.org/0000-0002-5321-9351 Faculdade Maurício de Nassau, Brasil E-mail: marillia_calado@hotmail.com

Débora Silva Amorim

ORCID: https://orcid.org/0000-0003-0894-9635 Unidade de Ensino Superior de Feira de Santana, Brasil E-mail: amorimdebora_@outlook.com

Flávia Nunes Fernandes

ORCID: https://orcid.org/0000-0002-0436-0797 Unidade de Ensino Superior de Feira de Santana, Brasil E-mail: fernandesnflavia@gmail.com

Roberta Amorim de Matos

ORCID: https://orcid.org/0000-0002-4655-4290 Unidade de Ensino Superior de Feira de Santana, Brasil E-mail: robertaamorim9@hotmail.com

Rafaela da Silva Moreira

ORCID: https://orcid.org/0000-0002-7772-1397 Unidade de Ensino Superior de Feira de Santana, Brasil E-mail: rafaelamoreira98s@ @otmail.com

Lilian de Lucena Oliveira

ORCID: https://orcid.org/0000-0002-4318-7253 Faculdade Maurício de Nassau, Brasil E-mail: lilianlucena22@hotmail.com

\begin{abstract}
Resumo
Objetivo: Analisar o perfil epidemiológico da tuberculose no estado de Pernambuco nos últimos 10 anos. Metodologia: Trata-se de um estudo descritivo, retrospectivo e quantitativo dos casos de tuberculose no estado de Pernambuco no período de 2009 a 2019. Os elementos utilizados são secundários do Sistema de Informação e Agravos de Notificação (SINAN) encontrados através do Departamento de Informática do Sistema Único de Saúde (DATASUS). Resultados: Nos últimos 10 anos foram notificados 61.449 casos de tuberculose no estado de Pernambuco. Ao analisar o perfil de casos confirmados, constatou-se que $70 \%$ são do sexo masculino, a faixa etária de 20 a 39 anos prevaleceu com 28.458 casos notificados. A forma clínica de tuberculose mais prevalente no período foi a pulmonar, com 52.242 casos notificados. Conclusão: Os resultados encontrados no presente estudo mostram que em Pernambuco houve prevalência da tuberculose em homens na idade reprodutiva e de baixa escolaridade. A tuberculose continua sendo um grave problema de saúde pública no Estado de Pernambuco. Portanto, ações diretas e indiretas voltadas à prevenção da tuberculose, em diversos espaços de regiões de saúde. Nesse sentido, compreender a dinâmica epidemiológica da tuberculose se torna prioritário a inclusão de ofertas de educação em saúde devido à falta de informação como os fatores de riscos relacionados à patologia de forma a evitar a infecção no estado de Pernambuco.
\end{abstract}

Palavras-chave: Tuberculose; Sistema de informação de saúde; Pernambuco; Epidemiologia; Saúde pública.

\begin{abstract}
Objective: To analyze the epidemiological profile of tuberculosis in the state of Pernambuco in the last 10 years. Methodology: This is a descriptive, retrospective and quantitative study of tuberculosis cases in the state of Pernambuco from 2009 to 2019. The elements used are secondary to the Information System and Notifiable Diseases (SINAN) found through the Department of Informatics of the Unified Health System (DATASUS). Results: In the last 10 years, 61,449 cases of tuberculosis were reported in the state of Pernambuco. When analyzing the profile of
\end{abstract}


confirmed cases, it was found that $70 \%$ are male, the age group 20 to 39 years old prevailed with 28,458 reported cases. The most prevalent clinical form of tuberculosis in the pulmonary period, with 52,242 reported cases. Conclusion: The results found in the present study show that in Pernambuco there was a prevalence of tuberculosis in men of reproductive age and with low education. Tuberculosis remains a serious public health problem in the State of Pernambuco. Therefore, direct and indirect actions aimed at preventing tuberculosis, in different areas of health regions. In this sense, understanding the epidemiological dynamics of tuberculosis becomes a priority for the inclusion of health education offers due to the lack of information such as the risk factors related to the pathology in order to avoid infection in the state of Pernambuco.

Keywords: Tuberculosis; Health information system; Pernambuco; Epidemiology; Public health.

\section{Resumen}

Objetivo: Analizar el perfil epidemiológico de la tuberculosis en el estado de Pernambuco en los últimos 10 años. Metodología: Se trata de un estudio descriptivo, retrospectivo y cuantitativo de los casos de tuberculosis en el estado de Pernambuco de 2009 a 2019. Los elementos utilizados son secundarios al Sistema de Información y Enfermedades Notificables (SINAN) encontrados a través del Departamento de Informática. del Sistema Único de Salud (DATASUS). Resultados: En los últimos 10 años, se notificaron 61.449 casos de tuberculosis en el estado de Pernambuco. Al analizar el perfil de los casos confirmados, se encontró que el $70 \%$ son hombres, predominó el grupo de edad de 20 a 39 años con 28.458 casos reportados. La forma clínica de tuberculosis más prevalente en el período pulmonar, con 52.242 casos notificados. Conclusión: Los resultados encontrados en el presente estudio muestran que en Pernambuco hubo una prevalencia de tuberculosis en hombres en edad reproductiva y con bajo nivel educativo. La tuberculosis sigue siendo un grave problema de salud pública en el estado de Pernambuco. Por ello, acciones directas e indirectas orientadas a la prevención de la tuberculosis, en diferentes áreas de las regiones sanitarias. En este sentido, comprender la dinámica epidemiológica de la tuberculosis se convierte en una prioridad para la inclusión de ofertas de educación en salud debido a la falta de información como los factores de riesgo relacionados con la patología para evitar la infección en el estado de Pernambuco.

Palabras clave: Tuberculosis; Sistema de información sanitaria; Pernambuco; Epidemiología; Salud pública.

\section{Introdução}

A tuberculose é uma doença infecciosa causada pela Mycobacterium tuberculosis, notado como um problema de saúde pública no Brasil. Embora que lesione especialmente os pulmões, esse agente infeccioso é capaz de causar doenças em quase todas as partes do corpo. A tuberculose é a responsável causa de morte por uma infecção em adultos no mundo (Lorran et al., 2021).

Frequentemente a infecção acontece por vias aéreas através do contato com espirros e tosse de pessoas portadoras do bacilo. Os primeiros sintomas dessa patologia é tosse persistente por mais de três semanas, com ou sem catarro, podendo apresentar febre no final da tarde por dias seguidos. Portanto, qualquer pessoa que apresentar os sintomas, deve procurar uma unidade de saúde na busca de um diagnóstico (Brasil, 2019). Estudos recentes monstraram que melhorias no acesso ao tratamento de tuberculose são necessários, primordialmente relacionados à realização de visita domiciliar e à distribuição do vale-transporte aos pacientes (Furlan; Marcon, 2017).

Essa micobactéria é caracterizada como um bacilo reto e possui a capacidade de sobreviver dentro de células fagocitárias. Devido a isso, ele é considerado um patógeno intracelular aeróbio estrito (De Lima et al., 2020). A infecção por Tuberculose é assintomática em pessoas com sistema imunológico competente dado que seu sistema imune opera formando uma barreira ao redor da bactéria. Os sintomas e sinais de tuberculose pulmonar (TBP) ativa são tosse, com expectoração (com ou sem sangue), dor no peito, fraqueza, perda de peso, febre e suores ao entardecer. A tuberculose é tratável com uso de antimicrobianos, sendo que o tratamento básico dura seis meses ou mais (WHO, 2018).

Segundo De Lima e autores (2020) existe uma extensa relação da tuberculose com pouca qualidade de vida, alimentação totalmente inadequada, falta de saneamento básico e o consumo exagerado de álcool e outras drogas. No entanto, o agravamento destas condições resulta em um aumento da vulnerabilidade, elevando um alto risco de contaminação nos indivíduos que vivem nessas condições. Devido à gravidade da doença, foi elaborado pelo Governo Federal o Programa 
Nacional de Controle da Tuberculose (PNCT), com intuito de reduzir a prevalência desse problema na população (BRASIL, 2018)

No Brasil a tuberculose é um importante problema de saúde pública, com cerca de 80 mil novos casos por ano e 5 mil mortes, posicionando o Brasil entre os 22 países com maior carga da doença (OPAS, 2020). Sua alta incidência e a relação direta com a pobreza permanece com a doença até os dias atuais como um importante problema de saúde pública (Costa, 2018).

De acordo com os dados descritos, este trabalho teve como objetivo traçar o perfil epidemiológico da tuberculose notificados no Sistema de Informação de Agravos de Notificação (SINAN), no período de 2009 a 2019 no estado de Pernambuco.

\section{Metodologia}

O corrente trabalho trata-se de um estudo ecológico, retrospectivo, descritivo com abordagem quantitativa (Pereira, et al., 2018) dos casos confirmados de tuberculose notificados em Pernambuco no período de 2009 a 2019. Os elementos utilizados são secundários do Sistema de Informação e Agravos de Notificação (SINAN) encontrados através do Departamento de Informática do Sistema Único de Saúde (DATASUS) na repartição de informações de saúde do Tabulador Genérico de Domínio Público (TABNET).

Com 98.311 km², Pernambuco é um dos 27 estados brasileiros. Localizado no centro leste da Região Nordeste, tem sua costa banhada pelo Oceano Atlântico. O estado faz limite com a Paraíba, Ceará, Alagoas, Bahia e Piauí. Também faz parte do território pernambucano, o arquipélago de Fernando de Noronha, a $545 \mathrm{~km}$ da costa. São 185 municípios - com um total de 8.796.032 habitantes - e tem a cidade do Recife como sua capital (Governo de Pernambuco, 2021).

Em 2011, foi definida as 04 Macrorregiões, em Resolução CIB/PE No 4086, de 11 de junho de 2018. Macrorregião 1 - Metropolitana (Regiões de saúde - I, II, III, XII); Macrorregião 2 - Agreste (Regiões de saúde - IV, V); Macrorregião 3 Sertão (Regiões de saúde - VI, X, XI) e Macrorregião 4 - Vale do São Francisco e Araripe - (Regiões de saúde -VII, VIIII e IX).

Figura 1 - Macrorregiões de Saúde do Estado de Pernambuco.

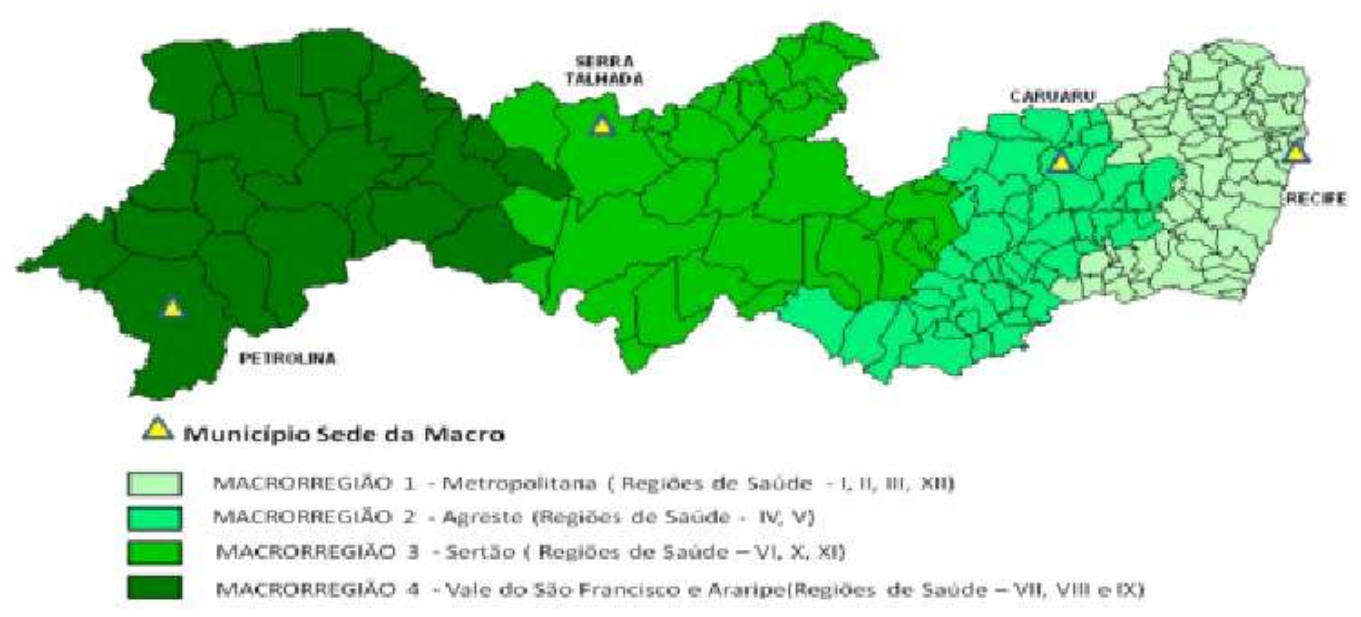

Fonte: Secretaria Estadual de Saúde de Pernambuco (2020). 
Foram criadas as 12 Gerências Regionais de Saúde (Geres), para apoiar todos os 184 municípios de Pernambuco mais a ilha de Fernando de Noronha. Cada uma dessas unidades administrativas da Secretaria Estadual de Saúde é responsável por uma parte das cidades, atuando de forma mais localizada na atenção básica, na reestruturação da rede hospitalar, nas ações municipais, no combate à mortalidade infantil e às diversas endemias (Secretaria Estadual de Saúde de Pernambuco, 2011).

Figura 2 - Regiões de Saúde do Estado de Pernambuco.

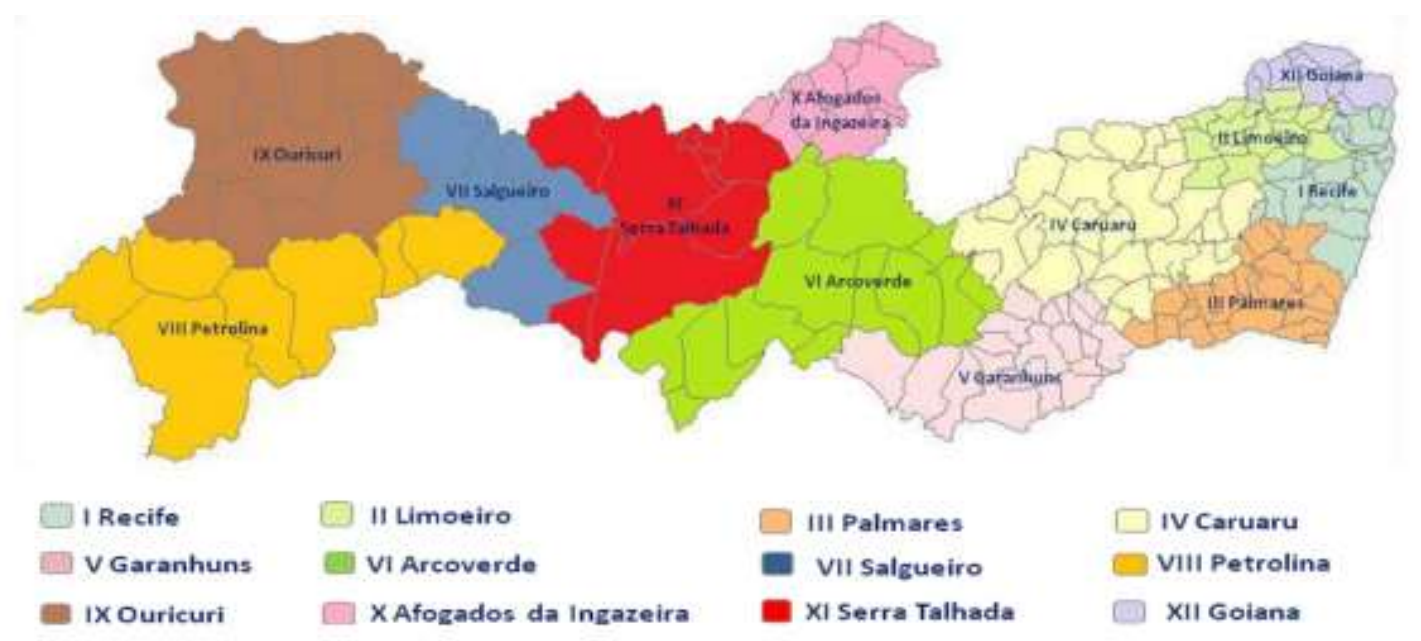

Fonte: Secretaria Estadual de Saúde de Pernambuco (2020).

Analisaram-se as variáveis: casos confirmados, macrorregião de saúde de residência, faixa etária, raça, sexo, escolaridade, zona de residência e forma clínica. Os resultados foram analisados de forma descritiva simples e organizados em gráficos no programa Microsoft Word Excel 2016 ®. A pesquisa de revisão de literatura foi realizada em bases de dados do Coordenação de Aperfeiçoamento de Pessoal de Nível Superior (CAPES), Scientific Eletronic Library Online (SCIELO) na Biblioteca Virtual em Saúde, tendo com os seguintes descritores "Tuberculose" e "Epidemiologia". Foram selecionados e revisados os artigos de maior relevância para o estudo e que apresentaram dados a ocorrência da patologia concomitantemente. Aplicando os critérios de exclusão respectivamente: estudos que apresentavam dados anteriores ao ano de 2015.

Devido a utilização somente de dados secundários, não houve necessidade de apreciação em Comitê de Ética em Pesquisa (CEP), estando de acordo com a Resolução de número 466 de 12 de dezembro de 2012 do Conselho Nacional de Saúde.

\section{Resultados e Discussão}

Nos últimos 10 anos foram notificados 61.449 casos de tuberculose no estado de Pernambuco. A situação epidemiológica é expressa nos gráficos a seguir. O Gráfico 1 demonstra a distribuição anual dos casos confirmados de tuberculose no estado de Pernambuco no período avaliado.

O Gráfico 1 demonstra o crescimento contínuo dos casos de tuberculose, 2019 (6.169), o ano que houve maior registro. Em 2009 (5.031) ocorreu o menor número de casos. 
Gráfico 1 - Casos confirmados de tuberculose segundo ano diagnóstico no estado de Pernambuco no período de 2009 a 2019.

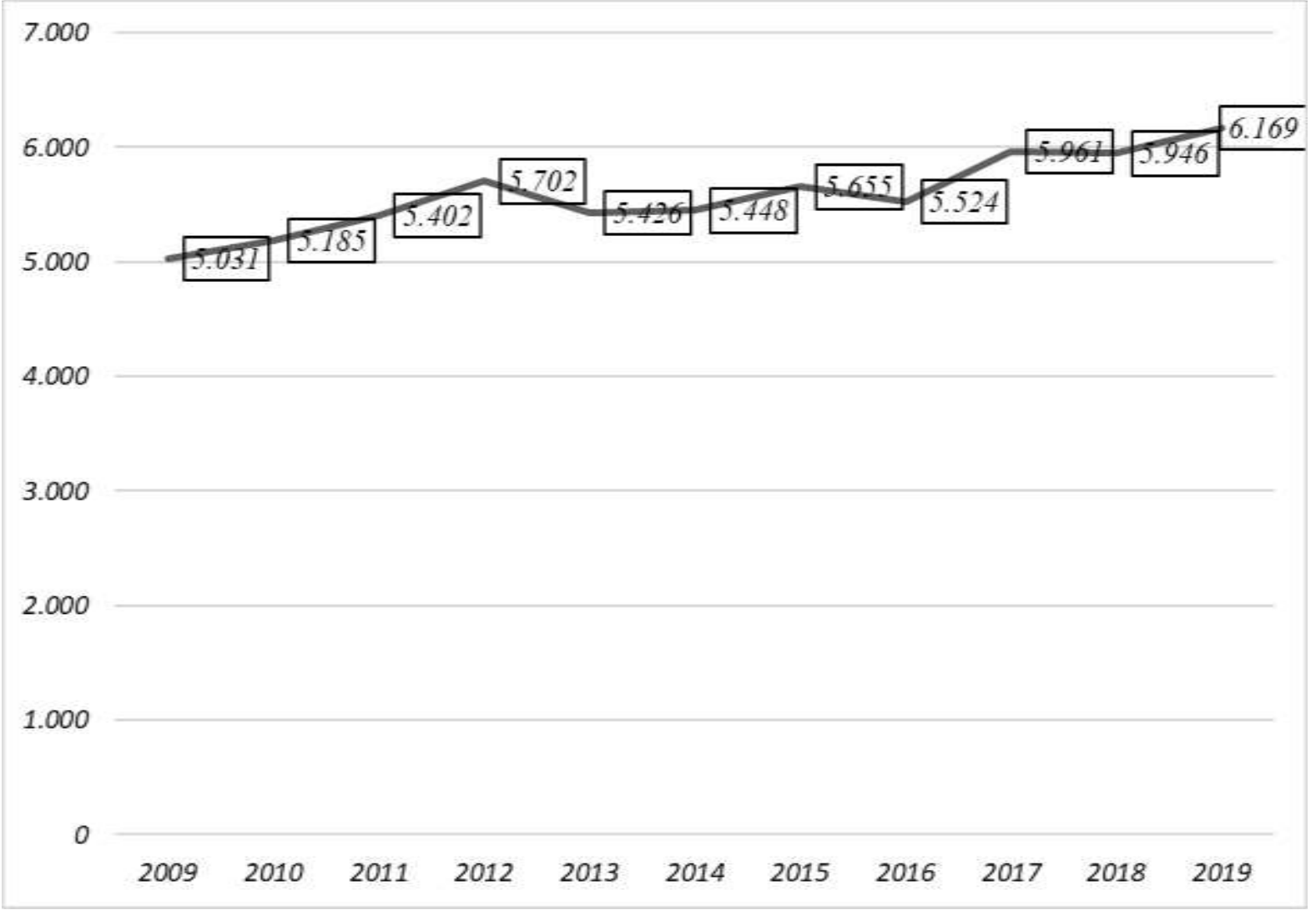

Fonte: Autoria própria (2021).

Em relação a localização dos casos por macrorregião de saúde de residência entre o período descrito, Vale do São Francisco e Araripe - 2.850 casos; Sertão - 2.010 casos; Metropolitana - 50.010 casos e Agreste - 6.180 casos, conforme observado na Tabela 1.

Tabela 1 - Casos confirmados de tuberculose por macrorregião de saúde de residência no período de 2009 a 2019.

\begin{tabular}{|c|c|}
\hline MACRORREGIÃO DE SAÚDE & NOTIFICAÇÃO \\
\hline Vale do S. Francisco e Araripe & 2.850 \\
\hline Sertão & 2.010 \\
\hline Metropolitana & 50.470 \\
\hline Agreste & 6.180 \\
\hline
\end{tabular}

Fonte: Autoria própria (2021).

De acordo com o Gráfico 2, ao analisar o sexo dos indivíduos com tuberculose, 70\% (42.726) casos correspondem ao sexo masculino, $30 \%$ (18.715) ao sexo feminino.

Para Santos e Martins, (2018), isso ocorre devido a maior exposição masculina às doenças infectocontagiosas por causa do tabagismo e alcoolismo, atrelada a resistência a prevenção de doenças por esse público. 
Gráfico 2 - Casos confirmados de tuberculose segundo o sexo no estado de Pernambuco no período de 2009 a 2019.

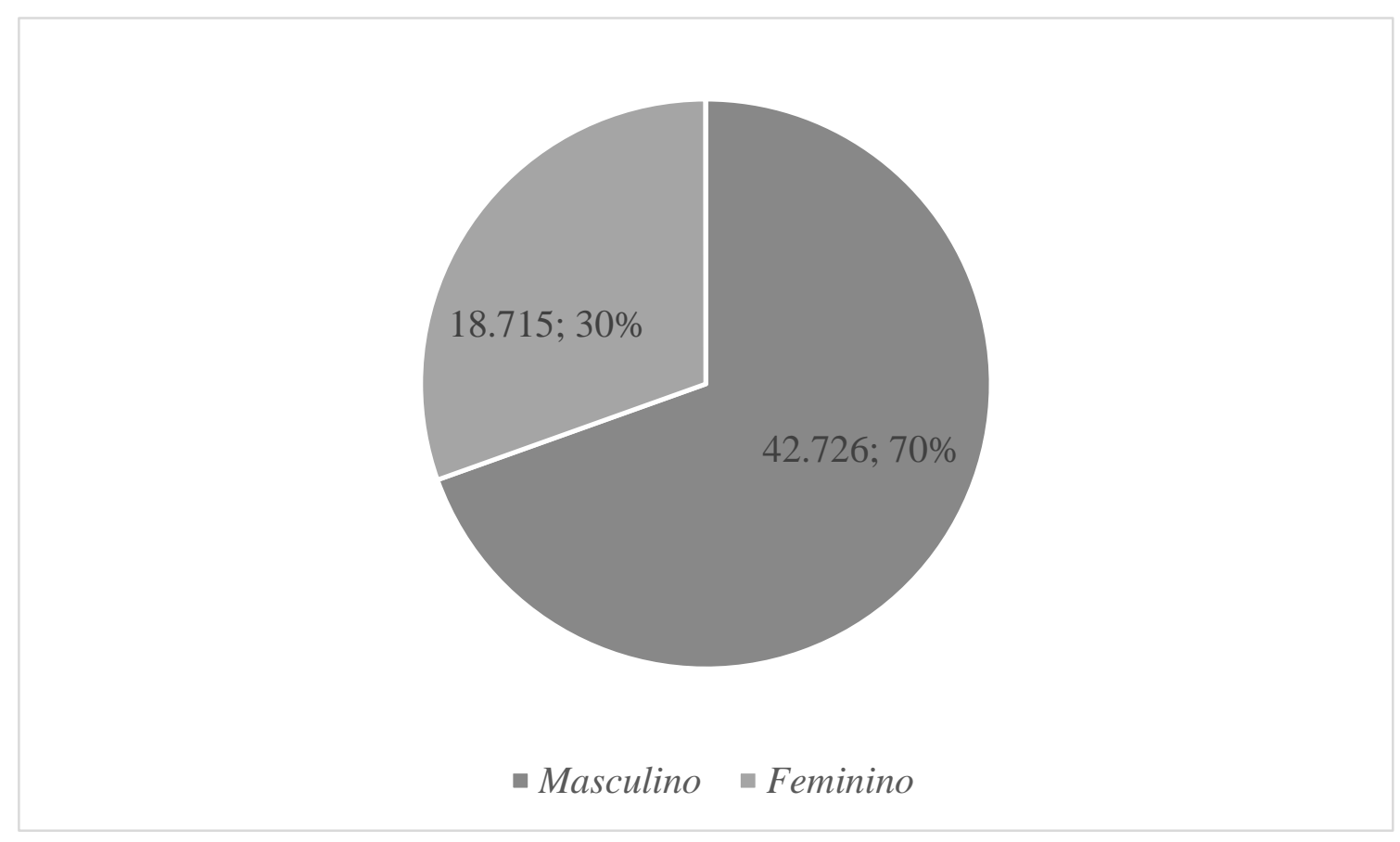

Fonte: Autoria própria (2021).

Freitas, et al. (2016), encontrou, em sua análise, evidências de que o sexo masculino é o mais afetado pela Tuberculose, legitimando com a literatura. Esta conjuntura pode ser legitimada devido o homem ter um perfil de mais divagação com a própria saúde aliado ao fato de estar mais exposto aos fatores de risco para a doença quando comparados às mulheres.

Averiguou-se as faixas etárias $<1$ a $80+$, foi possível observar que a faixa de maior prevalência de tuberculose nos anos avaliados foi de 20 a 39 anos, 28.458 dos casos, seguida dos indivíduos de 40 a 59 anos que representam 19.753. As pessoas de 60 a 79 anos somam 7.173 casos, as de 0 a 19 anos somam 4.588 casos e as 80 e + anos somam 1.008 casos (Gráfico 3). 
Gráfico 3 - Casos confirmados de tuberculose segundo a faixa etária no estado de Pernambuco no período de 2009 a 2019.

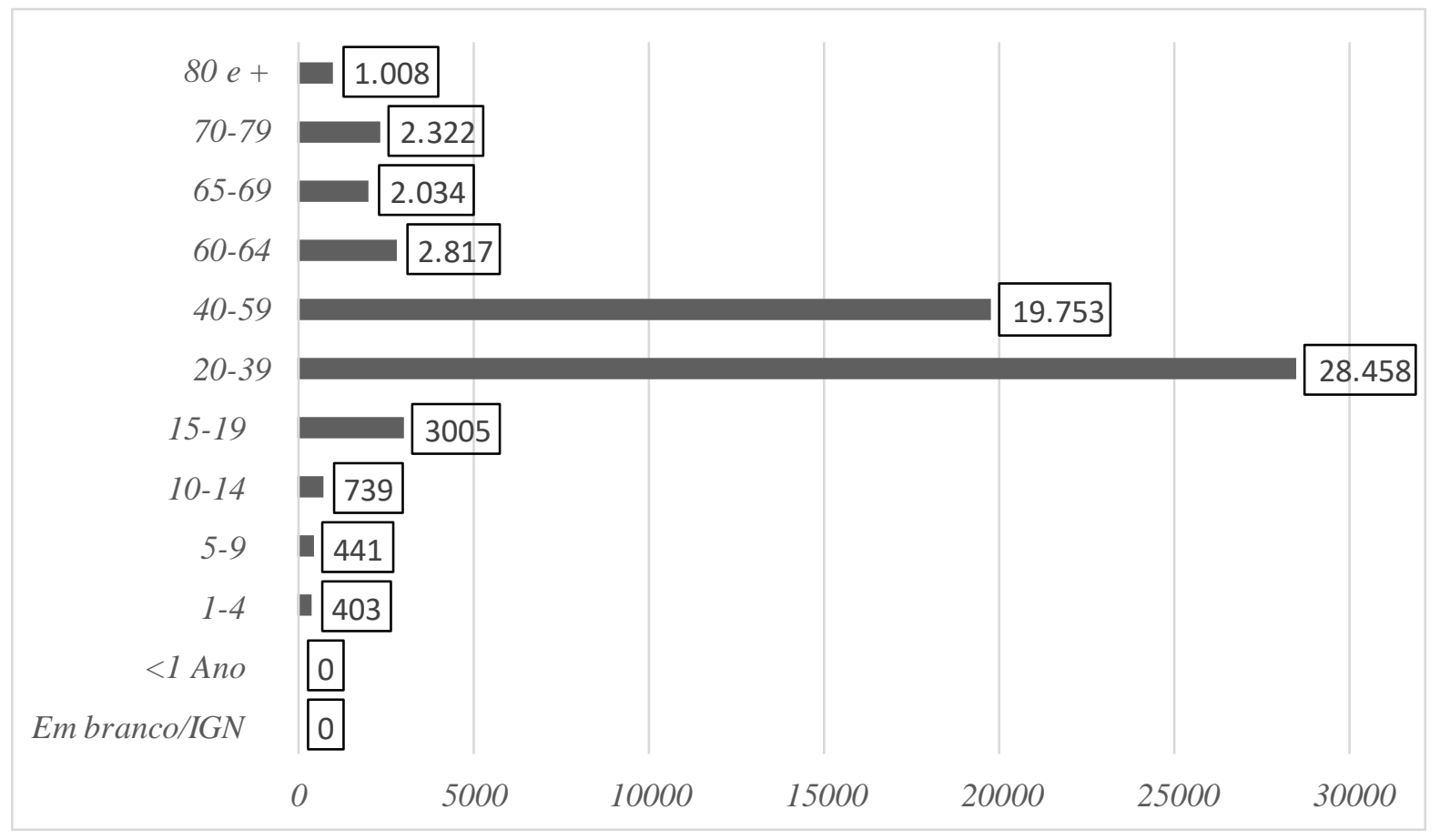

Fonte: Autoria própria (2021).

Com isso pode ser deslindado pela realidade de uma maior exibição às atividades realizadas nesta etapa da vida, como relações sexuais, transfusões de sangue com seringas e agulhas contaminadas, e uso de drogas injetáveis (Oliveira et al., 2018).

Em relação à escolaridade, 23.092 pessoas deixaram em branco essa informação na ficha, 3.668 pessoas marcaram analfabeto, 8.507 pessoas marcaram $1^{\mathrm{a}}$ a $4^{\mathrm{a}}$ série incompleta do ensino fundamental, 4.128 pessoas marcaram possuir $4^{\mathrm{a}}$ série completa do ensino fundamental, 8.510 pessoas marcaram $5^{\mathrm{a}}$ a $8^{\mathrm{a}}$ série incompleta do ensino fundamental, 2.738 pessoas marcaram ensino fundamental completo, 2.868 pessoas marcaram ensino médio incompleto, 4.868 pessoas marcaram ensino médio completo, 734 pessoas marcaram educação superior incompleta, 1.263 pessoas marcaram educação superior completa e 1.073 casos não se aplica. 
Gráfico 4 - Casos confirmados de tuberculose segundo a escolaridade no estado de Pernambuco no período de 2009 a 2019.

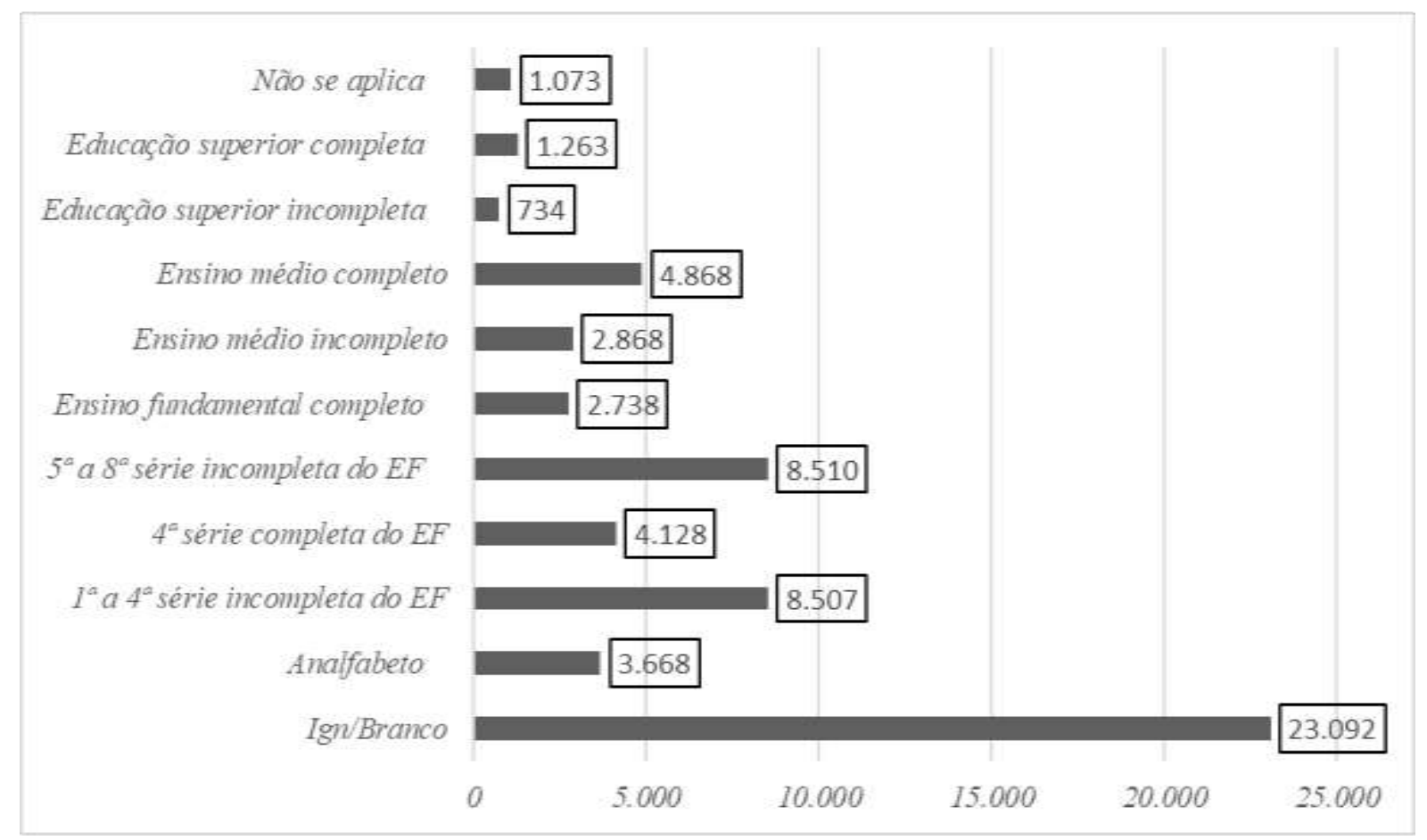

Fonte: Autoria própria (2021).

Neste estudo, cerca de 27.551 dos indivíduos com tuberculose eram analfabetos ou tinham como escolaridade o ensino fundamental, completo ou incompleto. Tais números sinalizam a veracidade de que, no Brasil, os cidadãos tipicamente marginalizados, de modo contínuo expostas a acometimento de doenças endêmicas, desemprego, desinformação, desnutrição de desestruturação dos serviços de saúde.

Analisando-se que entre os anos de 2009 a 2019 no estado de Pernambuco a zona de residência, Ignorado/branco 2.891, Urbana 51.495, Rural 6.418, Periurbana 645, nota-se que a zona urbana apresenta a maior porcentagem de notificações de casos de tuberculose no Estado de Pernambuco entre os anos 2009 a 2019 (Gráfico 5). 
Research, Society and Development, v. 10, n.3, e3710312916, 2021

(CC BY 4.0) | ISSN 2525-3409 | DOI: http://dx.doi.org/10.33448/rsd-v10i3.12916

Gráfico 5 - Casos confirmados de tuberculose segundo a zona de residência no estado de Pernambuco no período de 2009 a 2019.

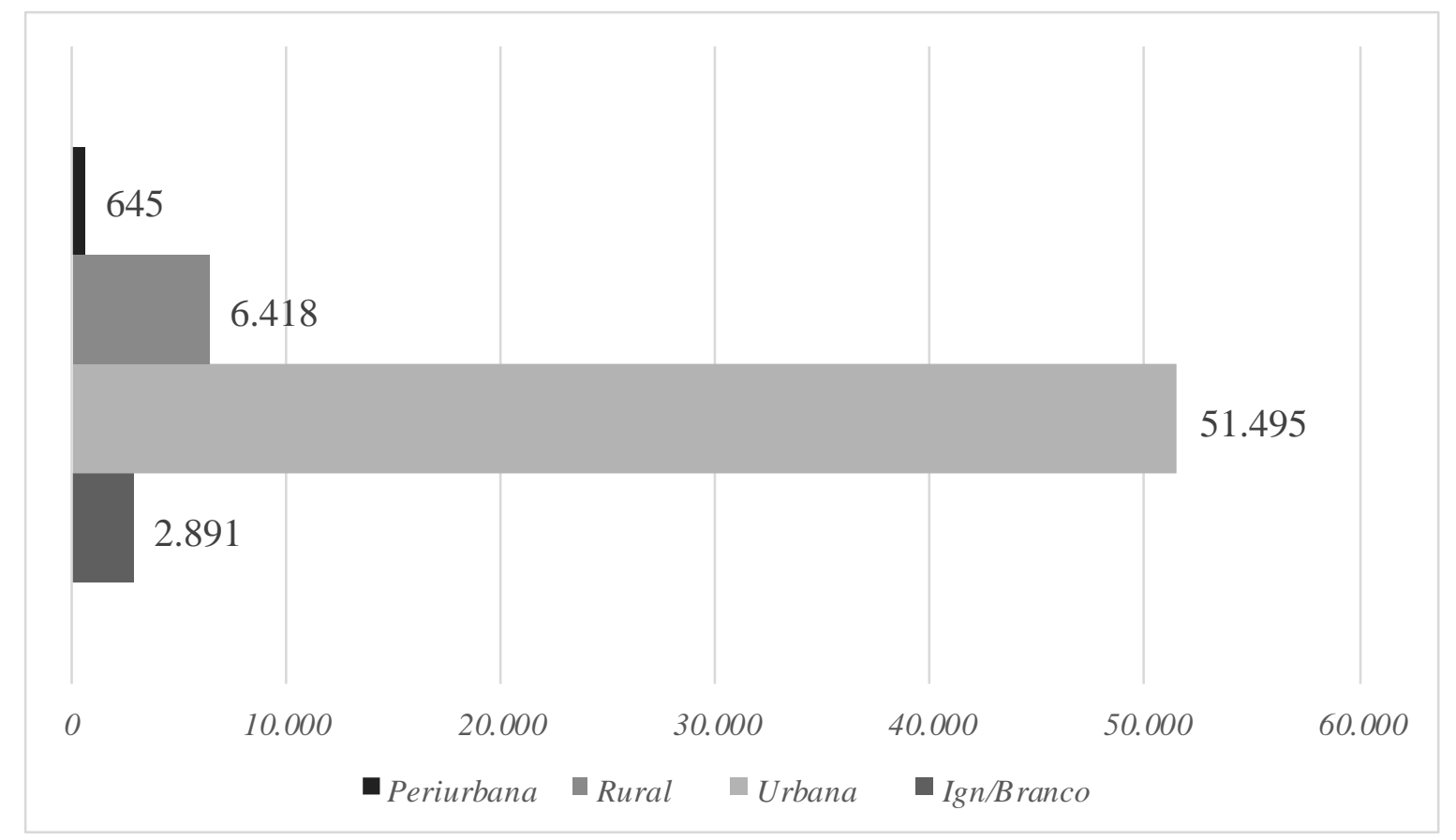

Fonte: Autoria própria (2021).

Conforme o Gráfico 6, é notório que a forma clínica de tuberculose mais prevalente no período foi a pulmonar 52.242 casos. A extrapulmonar 7.295 casos e a mista somente 1.904 casos. Como mostrado na primeira coluna do gráfico, a forma de apresentação da tuberculose não foi especificada em 8 casos confirmados (Ignorado/Branco). 
Research, Society and Development, v. 10, n.3, e3710312916, 2021

(CC BY 4.0) | ISSN 2525-3409 | DOI: http://dx.doi.org/10.33448/rsd-v10i3.12916

Gráfico 6 - Casos confirmados de tuberculose segundo a forma clínica no estado de Pernambuco no período de 2009 a 2019.

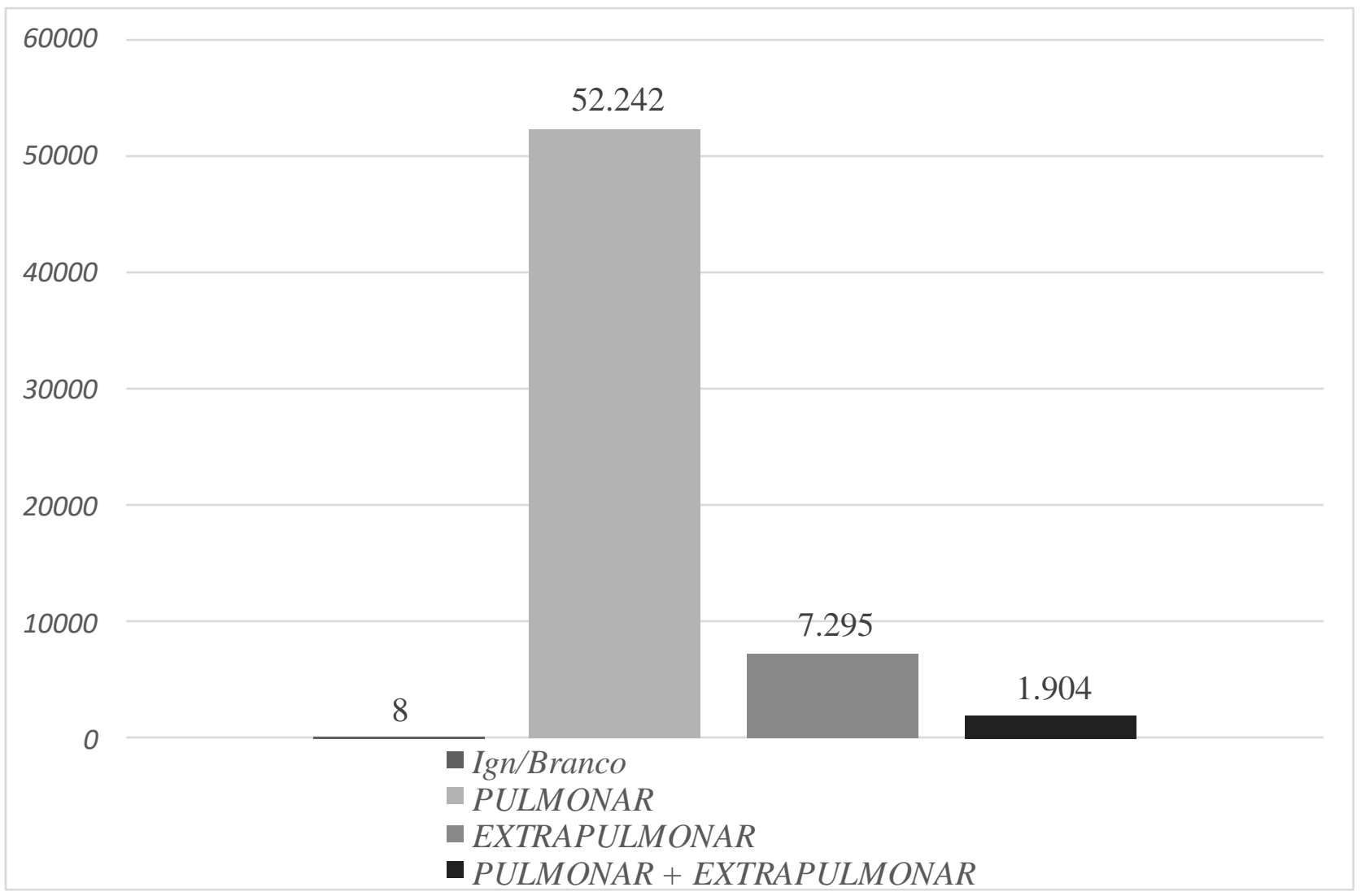

Fonte: Autoria própria (2021).

Em relação aos aspectos éticos, com base na cor/raça, os casos notificados da tuberculose entre o período analisado em Pernambuco, foram de 11.737 casos nos indivíduos de cor branca, pessoas de cor preta eram de 6.615 e indivíduos de cor parda eram de 35.331, foram aqueles que apresentaram o maior número de notificações (Gráfico 3). 
Gráfico 7 - Casos confirmados de tuberculose segundo raça no estado de Pernambuco no período de 2009 a 2019.

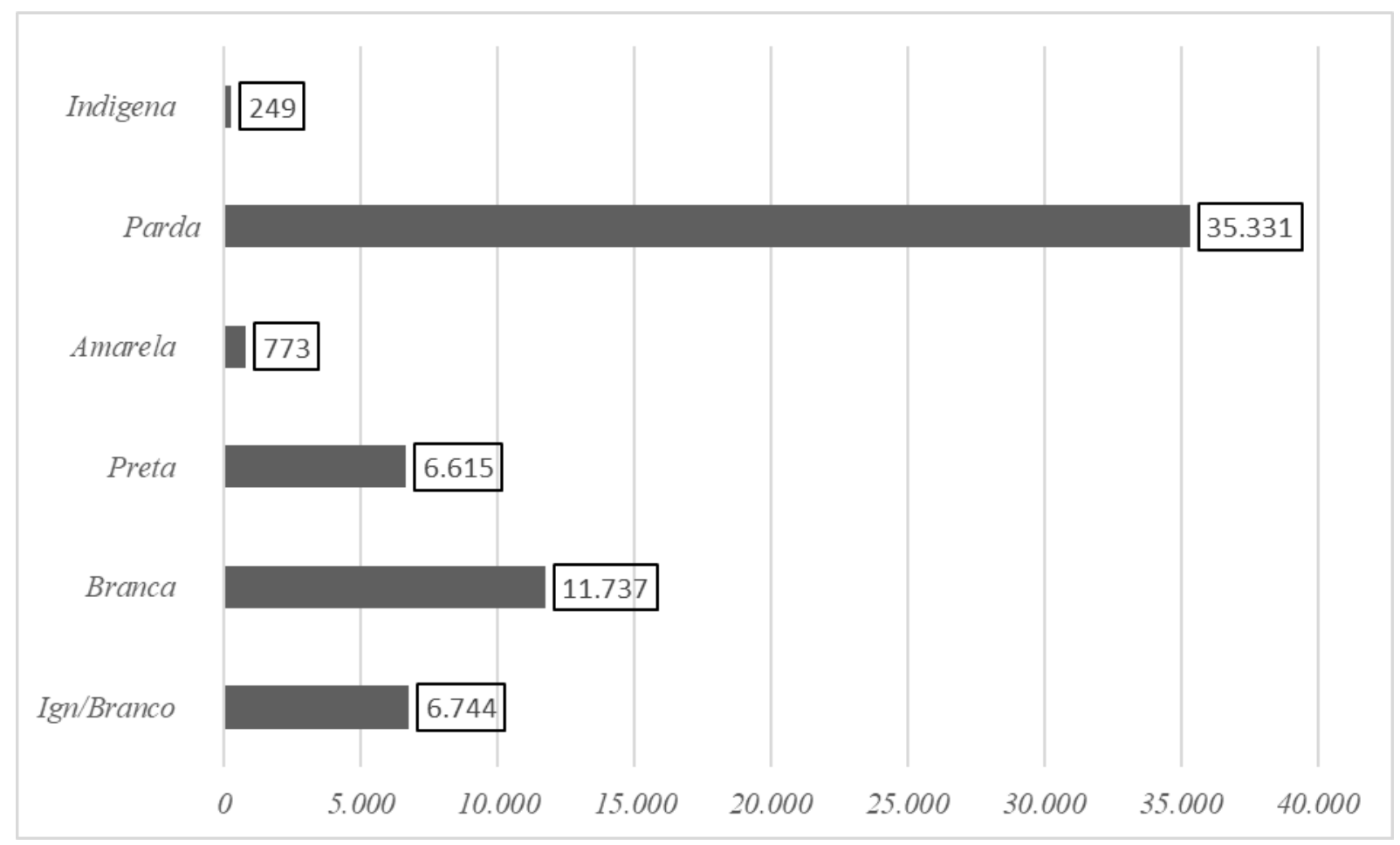

Fonte: Autoria própria (2021).

\section{Considerações Finais}

Os resultados obtidos nesta pesquisa possibilitaram conhecer as principais características dos casos notificados de tuberculose no estado de Pernambuco no período de 2009 a 2019.

A tuberculose continua sendo um grave problema de saúde pública no Estado de Pernambuco. Portanto, ações diretas e indiretas voltadas à prevenção da tuberculose, em diversos espaços de regiões de saúde. Nesse sentido, compreender a dinâmica epidemiológica da tuberculose se torna prioritário a inclusão de ofertas de educação em saúde devido à falta de informação como os fatores de riscos relacionados à patologia de forma a evitar a infecção no estado de Pernambuco. Como também a oferta de exames visando o diagnóstico precoce. Portanto a formulação de medidas eficazes para o controle dessa patologia que é tão relevante para o serviço público de saúde e epidemiológico. Sugerem-se a aplicação de mais estudos aprofundados devido à escassez sobre o assunto, cujo, engloba a doença, conforme o objetivo seja a inserção concreta de possíveis necessidades da população Pernambucana.

\section{Referências}

Costa, K. R. D. R. F. (2018). Análise do comportamento epidemiológico da tuberculose e suas relações com variáveis socioeconômicas no estado de Pernambuco.

de Lima, M. M. P., Ferreira, P. R. B., Batista, C. L., Magalhães, L. M., Souza, R. P., \& Oliveira, E. H. (2020). Análise temporal e epidemiológica dos casos de tuberculose no estado do Piauí, Brasil. Research, Society and Development, 9(2), e160922252-e160922252.

Freitas, W. M. T. M., Santos, C. C., Silva, M. M., \& Rocha, G. A. (2016). Perfil clínico-epidemiológico de pacientes portadores de tuberculose atendidos em uma unidade municipal de saúde de Belém, Estado do Pará, Brasil. Revista Pan-Amazônica de Saúde, 7(2), 45-50.

Furlan, M. C. R., \& Marcon, S. S. (2017). Avaliação do acesso ao tratamento de tuberculose sob a perspectiva de usuários. Cadernos Saúde Coletiva, 25(3), 339-347. Epub October 09, 2017. https://doi.org/10.1590/1414-462x201700030139 
Research, Society and Development, v. 10, n.3, e3710312916, 2021 (CC BY 4.0) | ISSN 2525-3409 | DOI: http://dx.doi.org/10.33448/rsd-v10i3.12916

Lorran, M., Lucas, D. S., Sutani, B., Carla, A., Silva, Túlio, M. S., Santos, M., Alves, M., Lucas G.M., \& Joyce C. A. (2021). Situação Atual Da Tuberculose No Estado De Pernambuco, Brasil. Scire Salutis, 11(1). http://sustenere.co/index.php/sciresalutis/article/view/4735

Ministério da Saúde. Secretaria de Vigilância em Saúde. (2020). Área técnica de Pneumologia Sanitária. Programa Nacional de Controle da Tuberculose. http://bvsms.saude.gov.br/bvs/publicacoes/ProgramaTB. pdf.

Ministério da Saúde. Secretaria de Vigilância em Saúde. Departamento de Vigilância das Doenças Transmissíveis. Manual de Recomendações para o Controle da Tuberculose no Brasil, Brasília.

Oliveira, L., Costa, C., Queiroz, A., de Araújo, T., Alves, A., de Sousa, K., \& Karina Reis, R. (2018). Análise epidemiológica da coinfecção tuberculose/hiv. Cogitare Enfermagem, 23(1). http://dx.doi.org/10.5380/ce.v23i1.51016

Organização Pan-Americana de Saúde (OPAS). (2018). 10 Principais Causas de Morte no Mundo. https:www.paho.or $\mathrm{g} / \mathrm{bra} /$ index.php?option=comcontent\&view=article $\& \mathrm{id}=5638: 10$ - principais-causas-de-morte-no-mundo\&Itemid=O

Pereira, A. S., Shitsuka, D. M., \& Parreira, F. J., Shitsuka, R. (2018). Metodologia da pesquisa científica. UFSM. https://repositorio.ufsm.br/bitstream/handle/1/15824/Lic_Computacao_Metodologia-Pesquisa-Cientifica.pdf?sequence=1

Santos, T. A. D., \& Martins, M. M. F. (2018). Perfil dos casos de reingresso após abandono do tratamento da tuberculose em Salvador, Bahia, Brasil. Cadernos Saúde Coletiva, 26(3), 233-240. https://doi.org/10.1590/1414-462x201800030235

Secretaria Estadual de Saúde de Pernambuco. (2011). Gerencias Regionais de Saúde. http://portal.saude.pe.gov.br/secretaria-executiva-de-coordenacaogeral/gerencias-regionais-de-saude

Secretaria Executiva de Gestão Estratégica e Participativa Diretoria Geral de Planejamento e Gestão Participativa. (2020). Plano Estadual de Saúde. http://portal.saude.pe.gov.br/sites/portal.saude.pe.gov.br/files/plano_estadual_de_saude_2020-2023.pdf

WHO (World Health Organization) (2020). Tuberculosis (TB). http://www.who.int/gho/tb/en/index.html\# 